\title{
Substance Independence of Efficiency of a Class of Heat Engines Undergoing Two Isothermal Processes
}

\author{
Y. Haseli \\ Department of Mechanical Engineering, Eindhoven University of Technology, P.O. Box 513, 5600 MB Eindhoven, The Netherlands \\ Correspondence should be addressed to Y. Haseli, y.haseli@tue.nl
}

Received 29 November 2010; Accepted 31 March 2011

Academic Editor: Y. Peles

Copyright () 2011 Y. Haseli. This is an open access article distributed under the Creative Commons Attribution License, which permits unrestricted use, distribution, and reproduction in any medium, provided the original work is properly cited.

Three power producing cycles have been so far known that include two isothermal processes, namely, Carnot, Stirling, and Ericsson. It is well known that the efficiency of the Carnot cycle represented by $1-T_{L} / T_{H}$ is independent of its working fluid. Using fundamental relationships between thermodynamic properties including Maxwell's relationships, this paper shows in a closed form that the Ericsson and the Stirling cycles also possess the Carnot efficiency irrespective of the nature of the working gas.

\section{Introduction}

A necessary step when teaching the second law in undergraduate thermodynamics classes is to introduce the concept of Carnot cycle, which was invented by Sadi Carnot, a French physicist and military engineer, in 1824. Historically, Carnot cycle has played a substantial role in further development of the second law of thermodynamics by Rudolf Clausius, a German physicist and mathematician, who in 1854 showed analytically that using an ideal gas as the working medium, the efficiency of the Carnot cycle is $1-T_{1} / T_{2}$ [1], where $T_{1}$ and $T_{2}$ denote the lowest and the highest temperatures of the cycle, respectively. This result led him to invent "entropy" in 1865 , and to derive his well-known inequality; that is, $\oint(Q / T) \leq 0$. Later on, these concepts were applied by several other pioneers such as Trevor [2], Kenrick [3], Kleeman [4], and Cantelo [5], to establish fundamental ideas. In these works, the Carnot cycle was treated as the regime of fully reversible, whereby satisfying $\oint(Q / T)=0$.

Under the assumption of working substance behaving as an ideal gas, the Carnot cycle is not the only one whose efficiency becomes $1-T_{1} / T_{2}$. Ericsson and Stirling cycles can also obtain the same efficiency [6]. These two cycles are similar to the Carnot cycle in a sense that they include two isothermal processes. However, the remaining two processes in the Ericsson cycle are isobaric while in the Stirling cycle they are isochoric. The $T-S$ and $P-V$ diagrams of these cycles are depicted in Figure 1. As in Carnot cycle, the Ericsson and the Stirling cycles would also satisfy $\oint(Q / T)=0$ at the regime of fully reversible.

Worthy of noting is that Stirling engine was invented by a Scottish engineer, Robert Stirling, in 1816; eight years before Carnot published his ideas in a 65-page book. In 1833, John Ericsson, an American Swedish-born inventor and mechanical engineer, patented the first Ericsson cycle, which is nowadays called Brayton cycle. The Ericsson cycle that we know today, with its idealized $T-S$ and $P-V$ diagrams shown in Figure 1, was invented in 1853. From a historical point of view, these three cycles which undergo isothermal compression and expansions processes, were designed in the same era. Nonetheless, only Carnot cycle has historically prevailed in the literature partly due to Benoit Clapeyron's efforts, another French engineer and physicist, who brought Carnot cycle to light by describing it in a graphical presentation in 1834, and mainly due to Clausius who formulated the first law and the second law on the basis of a Carnot cycle operating with an ideal gas.

As is well-known, the efficiency of Carnot engine is independent of the nature of the working fluid. The easiest proof is to refer to the corresponding $T-S$ diagram in Figure 1. The net entropy increase of the working fluid during the isothermal expansion process is equal to the net entropy decrease of the working medium in the isothermal compression process. This implies that $Q_{H} / T_{3}=Q_{L} / T_{1}$ 


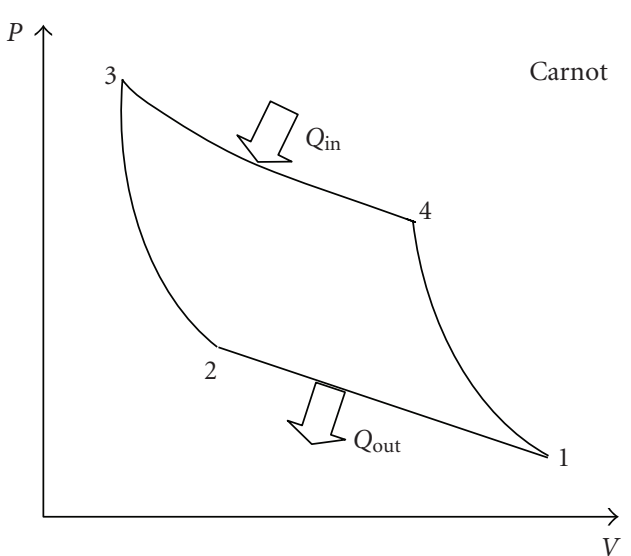

(a)

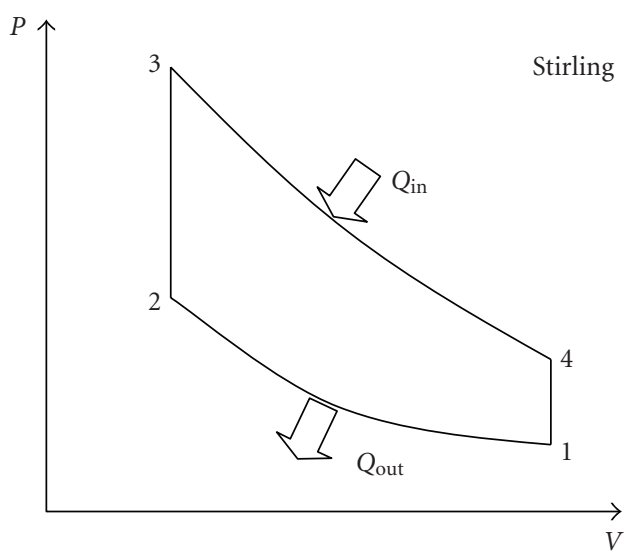

(c)

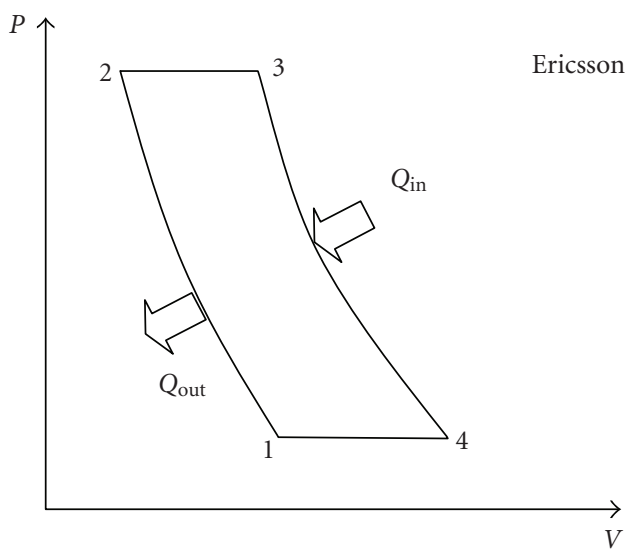

(e)

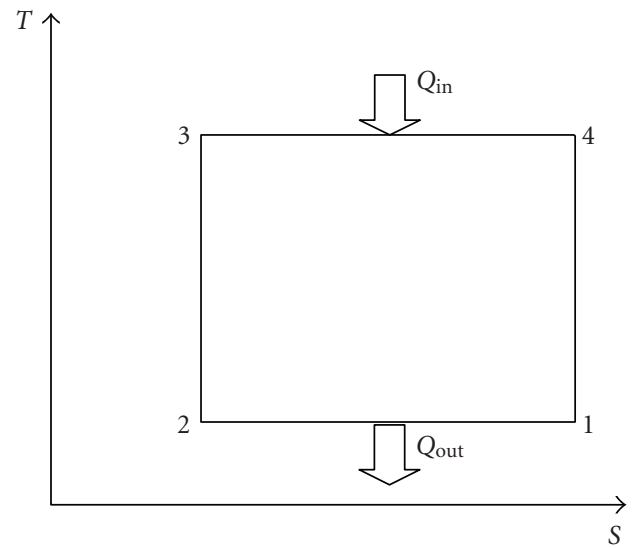

(b)

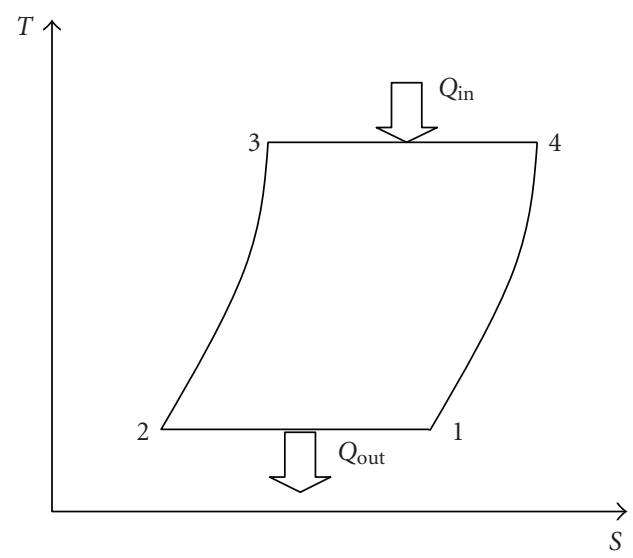

(d)

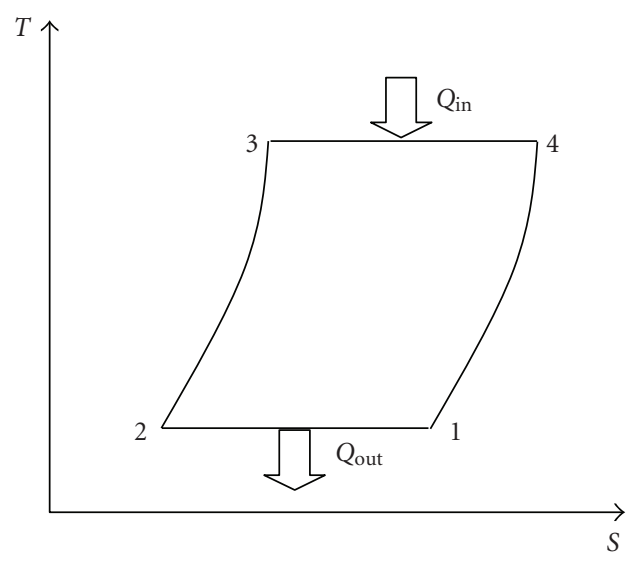

(f)

Figure 1: Pressure-volume and temperature-entropy diagrams of Carnot, Stirling, and Ericsson cycles.

which leads to an efficiency of $1-T_{1} / T_{3}$. The question which can be naturally asked is whether or not the other two competing cycles would still have the efficiency $1-T_{1} / T_{3}$ when operating with a working fluid other than the ideal gas; for instance with Van der Waals gas. This idea is followed as the main task of the present note; to investigate the efficiency of the idealized Stirling and Ericsson engines when operating with an arbitrary working gas. The analysis will be performed with the aid of the fundamental relationships between thermodynamic properties. Our starting point will be the following two well-established equations

$$
\begin{aligned}
& d U=T d S-P d V, \\
& d H=T d S+V d P .
\end{aligned}
$$


These equations are the consequence of the first law; that is, $d U=\delta Q-\delta W$. We will also need the following two relationships originally derived by Maxwell

$$
\begin{gathered}
\left(\frac{\partial S}{\partial V}\right)_{T}=\left(\frac{\partial P}{\partial T}\right)_{V}, \\
\left(\frac{\partial S}{\partial P}\right)_{T}=-\left(\frac{\partial V}{\partial T}\right)_{P} .
\end{gathered}
$$

The above relationships can be found in any standard thermodynamics textbook; for example, $[7,8]$.

\section{Stirling Cycle Operating with an Arbitrary Gas}

The ideal Stirling cycle (see Figure 1) consists of the following four processes: $1 \rightarrow 2$ isothermal compression which includes removal of heat from the system; $2 \rightarrow 3$ isochoric heat addition; $3 \rightarrow 4$ isothermal expansion with heat addition to the system; $4 \rightarrow 1$ isochoric heat rejection. To be able to precisely calculate the amount of heat transfer and work in various processes of the Stirling engine, we need to derive an equation for the internal energy $U$ which can be expressed as a function of temperature and volume; that is, $U=U(T, V)$. Taking into account the definition of the specific heat at constant volume $c_{V}$, the differential form of the internal energy can be expressed as

$$
d U=\left(\frac{\partial U}{\partial T}\right)_{V} d T+\left(\frac{\partial U}{\partial V}\right)_{T} d V=c_{V} d T+\left(\frac{\partial U}{\partial V}\right)_{T} d V .
$$

On the other hand, entropy $S$ can be expressed as a function of $T$ and $V$, too, with a differential form as

$$
d S=\left(\frac{\partial S}{\partial T}\right)_{V} d T+\left(\frac{\partial S}{\partial V}\right)_{T} d V=\left(\frac{\partial S}{\partial T}\right)_{V} d T+\left(\frac{\partial P}{\partial T}\right)_{V} d V
$$

Note that (3) is employed in (6). Substituting (6) into (1), we get

$$
d U=T\left(\frac{\partial S}{\partial T}\right)_{V} d T+\left[T\left(\frac{\partial P}{\partial T}\right)_{V}-P\right] d V
$$

It can be implied from (5) and (7) that

$$
T\left(\frac{\partial S}{\partial T}\right)_{V}=c_{V} d T
$$

Hence, (7) and (6) can be rewritten as

$$
\begin{gathered}
d U=c_{V} d T+\left[T\left(\frac{\partial P}{\partial T}\right)_{V}-P\right] d V, \\
d S=\frac{c_{V}}{T} d T+\left(\frac{\partial P}{\partial T}\right)_{V} d V .
\end{gathered}
$$

Applying $(\partial / \partial T)(\partial U / \partial V)=(\partial / \partial V)(\partial U / \partial T)$ to $(9)$ gives

$$
\left(\frac{\partial c_{V}}{\partial V}\right)_{T}=T\left(\frac{\partial^{2} P}{\partial T^{2}}\right)_{V}
$$

Integrating (11) at constant volume leads to a function for $c_{V}$ that is only dependent on temperature.

The entropy change of the working gas during the isochoric processes $2 \rightarrow 3$ and $4 \rightarrow 1$ can be evaluated using (10)

$$
\begin{aligned}
& S_{3}-S_{2}=\int_{2}^{3} \frac{c_{V}}{T} d T=F_{1}\left(T_{3}\right)-F_{1}\left(T_{2}\right) \quad F_{1}(T)=\int \frac{c_{V}}{T} d T, \\
& S_{1}-S_{4}=\int_{4}^{1} \frac{c_{V}}{T} d T=F_{1}\left(T_{1}\right)-F_{1}\left(T_{4}\right)=F_{1}\left(T_{2}\right)-F_{1}\left(T_{3}\right) .
\end{aligned}
$$

From (12) we conclude that

$$
S_{3}-S_{2}=S_{4}-S_{1} \quad \text { or } \quad S_{1}-S_{2}=S_{4}-S_{3}
$$

Applying the first law to the heat addition process $2 \rightarrow 3$ while taking into account (9), we get

$$
\begin{aligned}
Q_{23} & =\int_{2}^{3} d U=\int_{2}^{3} c_{V} d T \\
& =F_{2}\left(T_{3}\right)-F_{2}\left(T_{2}\right),
\end{aligned}
$$

where $F_{2}(T)=\int c_{V} d T$.

Likewise, the amount of heat rejection during process $4 \rightarrow 1$ is determined as

$$
Q_{41}=F_{2}\left(T_{1}\right)-F_{2}\left(T_{4}\right)=F_{2}\left(T_{2}\right)-F_{2}\left(T_{3}\right) .
$$

Hence, the amount of heat addition during the isochoric process $2 \rightarrow 3$ is the same as the amount of heat rejected during the isochoric process $4 \rightarrow 1$. The amount of heat removed during process $4 \rightarrow 1$ can be used to meet the heat requirement of process $2 \rightarrow 3$.

In a similar manner, we can determine the entropy change and the amount of heat addition/rejection during the isothermal processes $3 \rightarrow 4$ and $1 \rightarrow 2$. Using (10) for the isothermal processes, we have

$$
\begin{aligned}
& S_{4}-S_{3}=\int_{3}^{4}\left(\frac{\partial P}{\partial T}\right)_{V} d V \\
& S_{2}-S_{1}=\int_{1}^{2}\left(\frac{\partial P}{\partial T}\right)_{V} d V .
\end{aligned}
$$

From (13) and (16), we conclude that

$$
\left|\int_{3}^{4}\left(\frac{\partial P}{\partial T}\right)_{V} d V\right|=\left|\int_{1}^{2}\left(\frac{\partial P}{\partial T}\right)_{V} d V\right|
$$

The heat addition/rejection during process $3 \rightarrow 4 / 1 \rightarrow 2$ can be obtained by applying the first law

$$
\begin{aligned}
\delta Q & =d U+P d V \\
& =c_{V} d T+\left[T\left(\frac{\partial P}{\partial T}\right)_{V}-P\right] d V+P d V \\
& =T\left(\frac{\partial P}{\partial T}\right)_{V} d V
\end{aligned}
$$


Hence,

$$
\begin{aligned}
& Q_{12}=T_{1} \int_{1}^{2}\left(\frac{\partial P}{\partial T}\right)_{V} d V, \\
& Q_{34}=T_{3} \int_{3}^{4}\left(\frac{\partial P}{\partial T}\right)_{V} d V .
\end{aligned}
$$

Recalling that the amount of heat addition in process $2 \rightarrow 3$ equals the amount of heat rejection in process $4 \rightarrow 1$, the efficiency of the Stirling cycle is determined as follows:

$$
\eta_{S}=1-\frac{Q_{12}}{Q_{34}} .
$$

Substituting (19) and (20) into (21) and taking into account (17), we finally find

$$
\eta_{S}=1-\frac{T_{1}}{T_{3}} .
$$

\section{Ericsson Cycle Operating with an Arbitrary Gas}

The ideal Ericsson cycle (see Figure 1) includes the following four processes: $1 \rightarrow 2$ isothermal compression which includes removal of heat from the system; $2 \rightarrow 3$ isobaric heat addition; $3 \rightarrow 4$ isothermal expansion and heat addition to the system; $4 \rightarrow 1$ isobaric heat rejection. We need to take the same procedure outlined in the preceding section to determine the thermal efficiency of the Ericsson cycle. However, in this section, we will employ the definition of enthalpy for the analysis of the Ericsson cycle. For this purpose, suppose that enthalpy $H$ can be expressed as a function of temperature and pressure; that is, $H=H(T, P)$, or in a differential form as

$$
d H=\left(\frac{\partial H}{\partial T}\right)_{P} d T+\left(\frac{\partial H}{\partial P}\right)_{T} d P=c_{P} d T+\left(\frac{\partial H}{\partial V}\right)_{T} d V .
$$

We further suppose that entropy $S$ can be expressed as a function of $T$ and $P$ with a differential form as below in which (4) is employed.

$$
d S=\left(\frac{\partial S}{\partial T}\right)_{P} d T+\left(\frac{\partial S}{\partial P}\right)_{T} d P=\left(\frac{\partial S}{\partial T}\right)_{P} d T-\left(\frac{\partial V}{\partial T}\right)_{P} d P
$$

Substituting (24) into (2) yields

$$
d H=T\left(\frac{\partial S}{\partial T}\right)_{P} d T+\left[V-T\left(\frac{\partial V}{\partial T}\right)_{P}\right] d P .
$$

From (23) and (25), we conclude that

$$
T\left(\frac{\partial S}{\partial T}\right)_{P}=c_{P} d T .
$$

Hence,

$$
d H=c_{P} d T+\left[V-T\left(\frac{\partial V}{\partial T}\right)_{P}\right] d P .
$$

Eliminating $d H$ between (27) and (2) and rearranging the resulting expression for $S$ gives

$$
d S=\frac{c_{P} d T}{T}-\left(\frac{\partial V}{\partial T}\right)_{P} d P .
$$

Applying $(\partial / \partial T)(\partial H / \partial P)=(\partial / \partial P)(\partial H / \partial T)$ to $(27)$ yields

$$
\left(\frac{\partial c_{P}}{\partial P}\right)_{T}=-T\left(\frac{\partial^{2} V}{\partial T^{2}}\right)_{P}
$$

Equation (29) implies that $c_{P}$ at an isobaric process is only a function of temperature. So, the entropy change of the working substance during the isobaric processes $2 \rightarrow 3$ and $4 \rightarrow 1$ can be evaluated using (28) as follows:

$$
\begin{aligned}
& S_{3}-S_{2}=\int_{2}^{3} \frac{c_{P}}{T} d T=F_{3}\left(T_{3}\right)-F_{3}\left(T_{2}\right) \quad F_{3}(T)=\int \frac{c_{P}}{T} d T, \\
& S_{1}-S_{4}=\int_{4}^{1} \frac{c_{P}}{T} d T=F_{3}\left(T_{1}\right)-F_{3}\left(T_{4}\right)=F_{3}\left(T_{2}\right)-F_{3}\left(T_{3}\right) .
\end{aligned}
$$

It can be inferred from (3) that the entropy increase during process $2 \rightarrow 3$ is equal to the entropy decrease in process $4 \rightarrow 1$. Hence,

$$
S_{1}-S_{2}=S_{4}-S_{3} .
$$

The amount of heat addition during isobaric process $2 \rightarrow 3$ can be obtained using the first law as follows.

$$
Q_{23}=\int_{2}^{3} d H=\int_{2}^{3} c_{P} d T=F_{4}\left(T_{3}\right)-F_{4}\left(T_{2}\right),
$$

where $F_{4}(T)=\int c_{P} d T$.

Likewise, the amount of heat rejection during isobaric process $4 \rightarrow 1$ is determined as

$$
Q_{41}=F_{4}\left(T_{1}\right)-F_{4}\left(T_{4}\right)=F_{4}\left(T_{2}\right)-F_{4}\left(T_{3}\right) .
$$

It can be implied from (32) and (33) that $Q_{23}=-Q_{41}$. So, the amount of heat rejected during process $4 \rightarrow 1$ can be bypassed to meet the heat requirement of process $2 \rightarrow 3$.

The change in entropy during the isothermal processes $3 \rightarrow 4$ and $1 \rightarrow 2$ is evaluated with the aid of (28)

$$
\begin{aligned}
& S_{4}-S_{3}=-\int_{3}^{4}\left(\frac{\partial V}{\partial T}\right)_{P} d P, \\
& S_{2}-S_{1}=-\int_{1}^{2}\left(\frac{\partial V}{\partial T}\right)_{P} d P .
\end{aligned}
$$

A combination of (31) and (34) leads to

$$
\left|\int_{3}^{4}\left(\frac{\partial V}{\partial T}\right)_{P} d P\right|=\left|\int_{1}^{2}\left(\frac{\partial V}{\partial T}\right)_{P} d P\right| .
$$

The heat addition/rejection during process $3 \rightarrow 4 / 1 \rightarrow 2$ can be obtained by applying the first law

$$
\begin{aligned}
\delta Q & =d H-V d P \\
& =c_{P} d T+\left[V-T\left(\frac{\partial V}{\partial T}\right)_{P}\right] d P-V d P \\
& =-T\left(\frac{\partial V}{\partial T}\right)_{P} d P .
\end{aligned}
$$


Hence,

$$
\begin{aligned}
& Q_{12}=-T_{1} \int_{1}^{2}\left(\frac{\partial V}{\partial T}\right)_{P} d P, \\
& Q_{34}=-T_{3} \int_{3}^{4}\left(\frac{\partial V}{\partial T}\right)_{P} d P .
\end{aligned}
$$

Finally, taking into account (35) the efficiency of the Ericsson cycle is obtained as follows

$$
\begin{aligned}
\eta_{E} & =1-\frac{Q_{12}}{Q_{34}}=1-\frac{-T_{1} \int_{1}^{2}(\partial V / \partial T)_{P} d P}{-T_{3} \int_{3}^{4}(\partial V / \partial T)_{P} d P} \\
& =1-\frac{T_{1}}{T_{3}} .
\end{aligned}
$$

\section{Discussion}

The important aspect of the results presented in this note is that the Carnot cycle is not the only one which possesses the highest efficiency among engines operating between the same low- and high-temperature thermal reservoirs. Indeed, the idealized Ericsson and Stirling engines are as efficient as the Carnot engine. These results reveal that the class of heat engines which undergo two isothermal processes has the same efficiency irrespective of the nature of the working substance. So, the Ericsson and the Stirling cycles would deserve to be introduced in thermodynamics classrooms and textbooks; especially considering their practical importance. The Ericsson cycle is the limiting design of a gas turbine engine operating on the basis of a Brayton cycle with multiple intercooler and reheater, whereas the Stirling cycle is the ideal design of a recuperating external combustion engine. Both reversible and irreversible configurations of the Ericsson and the Stirling engines have received tremendous attention in the literature. Among many scholars researching in these fields, the contribution of Organ $[9,10]$ who has been continuously working on Stirling engines is acknowledgeable.

Referring to the available historical evidences, one would realize why the Carnot cycle, among the group of heat engines with two isothermal processes, has prevailed in the literature as the most efficient one. This is due to firstly Sadi Carnot's book in which he attempted to prove by means of rational reasoning (in his era neither the first law nor the second law had been fully realized and formulated) that only his proposed engine would produce a maximum work from a given amount of heat [11], even though his ideas were remained unnoticed for a decade. On the other hand, Clapeyron played a key role by representing the Carnot's cycle graphically in a $P-V$ diagram such as shown in Figure 1. More importantly, Carnot's rationalized theorems together with Clapeyron's work allowed Clausius to further advance borders of thermodynamics by formulating the first and the second laws on the basis of the Carnot cycle.

Worthy of further discussion is performance of the Ericsson and the Stirling engines when exchanging finite time heat with given high and low temperature reservoirs. In 1975 Curzon and Ahlborn [12] criticized that a Carnot cycle undergoing infinitesimal processes would physically result in zero power output. They proposed a model of endoreversible Carnot cycle exchanging finite time heat with thermal reservoirs, and showed that the efficiency at maximum power operation obeys $\eta_{\mathrm{CA}}=1-\left(T_{L} / T_{H}\right)^{0.5}$, with $T_{L}$ and $T_{H}$ representing the temperatures of thermal reservoirs. Either of the Stirling cycle or the Ericsson cycle could also operate with the above efficiency at maximum power condition. The key part of the Curzon-Aholborn's demonstration is to employ $Q_{12} / Q_{34}=T_{1} / T_{3}$ (referring to the nomenclature of Figure 1). As was shown in the present note, the efficiency of the Stirling and the Ericsson cycles obeys $1-T_{1} / T_{3}$. Thus, it can be demonstrated with the same methodology of Curzon-Aholborn [12] that the efficiency of these cycles would be $\eta_{\mathrm{CA}}=1-\left(T_{L} / T_{H}\right)^{0.5}$ at maximum power operation.

\section{Acknowledgment}

The author would like to thank the reviewers for performing a careful review.

\section{References}

[1] I. Müller, A History of Thermodynamics: The Doctrine of Energy and Entropy, Springer, New York, NY, USA, 2007.

[2] J. E. Trevor, "The exposition of the entropy theory," Journal of Physical Chemistry, vol. 4, no. 6, pp. 514-528, 1900.

[3] F. B. Kenrick, "A mechanical model to illustrate the gas laws," Journal of Physical Chemistry, vol. 8, no. 5, pp. 351-356, 1904.

[4] R. D. Kleeman, "The absolute zero of the controllable entropy and internal energy of a substance or mixture," Journal of Physical Chemistry, vol. 31, no. 5, pp. 747-756, 1927.

[5] R. C. Cantelo, "The second law of thermodynamics in chemistry," Journal of Physical Chemistry, vol. 32, no. 7, pp. 982-989, 1928.

[6] A. M. Y. Razak, Industrial Gas Turbines: Performance and Operability, Taylor \& Francis, Boca Raton, Fla, USA, 2003.

[7] G. van Wylen, R. Sonntag, and C. Borgnakke, Fundamental of Classical Thermodynamics, John Wiley \& Sons, New York, NY, USA, 1994.

[8] A. Bejan, Advanced Engineering Thermodynamics, John Wiley \& Sons, New York, NY, USA, 1997.

[9] A. J. Organ, The Regenerator and Stirling Engine, John Wiley \& Sons, New York, NY, USA, 1997.

[10] A. J. Organ, The Air Engine: Stirling Cycle Power for a Sustainable Future, CRC Press, 2007.

[11] R. H. Thurston, Ed., Reflections on the Motive Power of Heat, John Wiley \& Sons, New York, NY, USA, 2nd edition, 1897.

[12] F. L. Curzon and B. Ahlborn, "Efficiency of a carnot engine at maximum power output," American Journal of Physics, vol. 43, no. 1, pp. 22-24, 1975. 

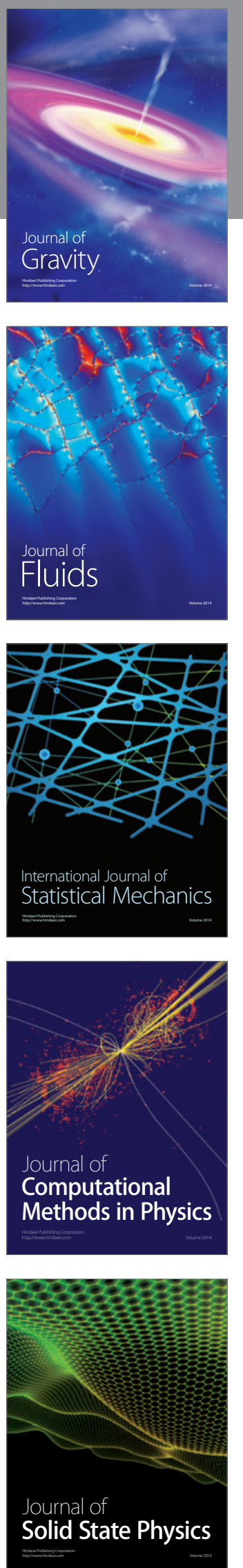

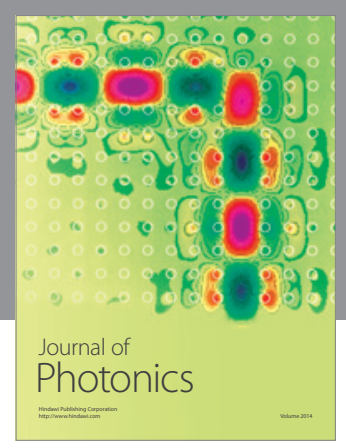

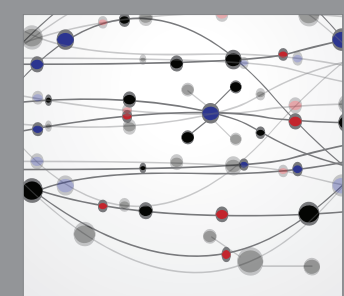

The Scientific World Journal
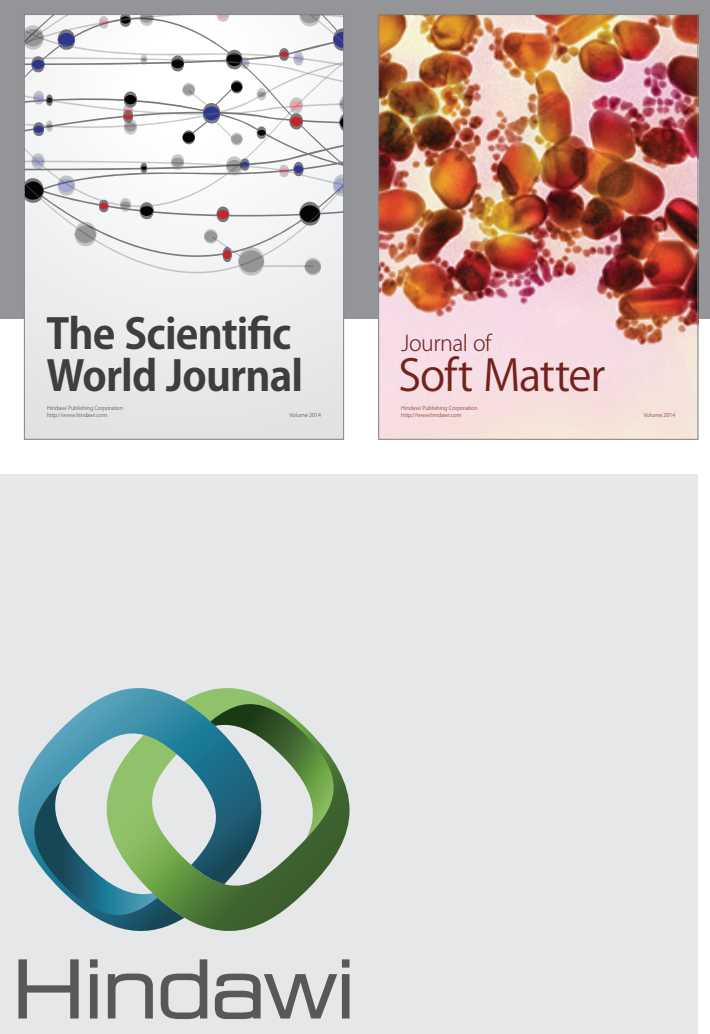

Submit your manuscripts at

http://www.hindawi.com
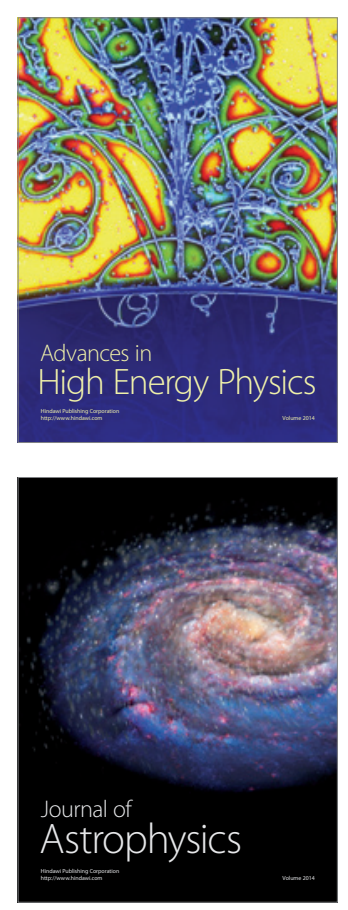
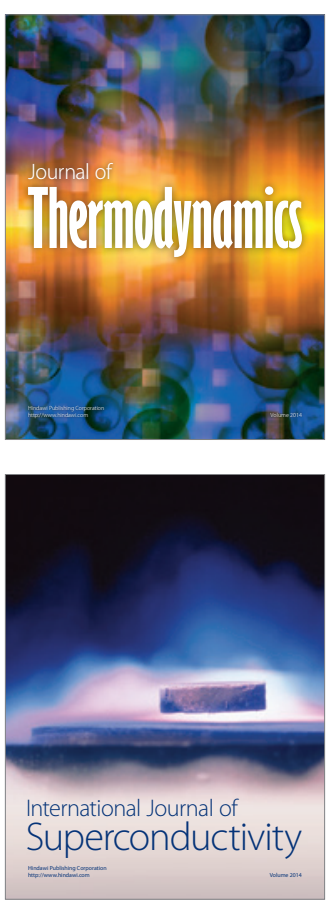
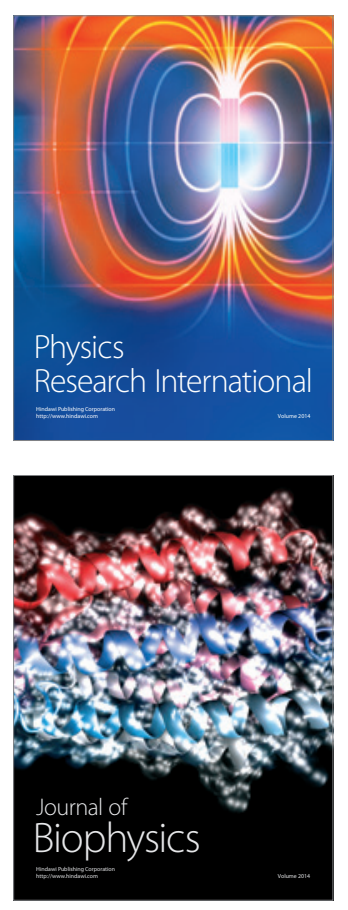
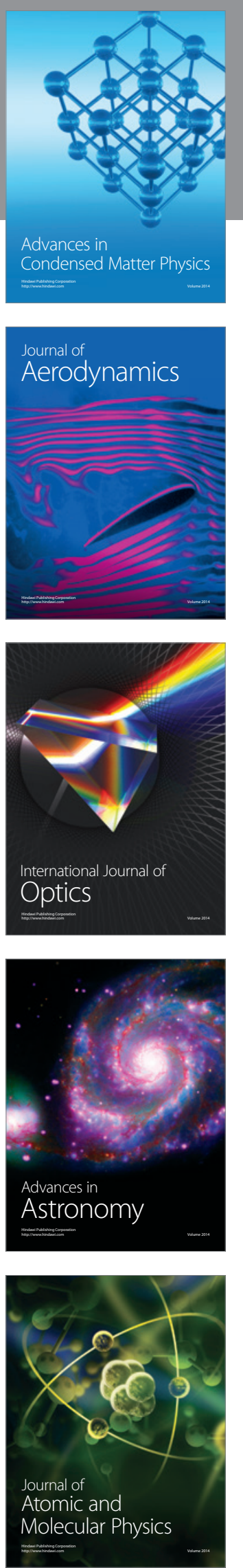